\title{
APROXIMACIÓN REgIONAL DEL TURISMO ALTERNATIVO: DEL CASO DE ESTUDIO A LA MODELIZACIÓN
}

\author{
Samuel Jouault \\ samuel.jouault@correo.uady.mx \\ Facultad de Ciencias Antropológicas \\ Universidad Autónoma de Yucatán \\ Centro de Estudios Mexicanos \\ y Centroamericanos
}

La región, noción esencial de la geografía, pero también polisémica e imprecisa, califica cualquier espacio delimitado y por consecuencia espacialmente ubicado, con significación objetiva y colectiva. Así, la geografía regional estudia las regiones; es decir, espacios que presentan alguna homogeneidad constitutiva, tal como la actividad turística. Es el caso de la región del traspaís de Cancún-Riviera Maya, la cual conoce desde hace quince años una gran efervescencia turística. En numerosos pueblos mayas de esta región las sociedades locales han apostado al turismo como actividad económica complementaria o principal. A través de los juegos de escalas, se abordará la importancia de la regionalización, no únicamente en el estudio y comprensión del turismo alternativo, sino a través de la modelización basada en el papel que los actores juegan en la turistificación de los espacios rurales de una región determinada.

Palabras clave: Cancún-Riviera Maya, geografía regional, modelo, región turística, turistificación.

\section{REGIONAL APPROACH OF ALTERNATIVE TOURISM: FROM CASE STUDY TO MODELING}

The region, as an essential notion of Geography, is a polysemic and imprecise term that qualifies any defined space which is spatially located and possesses an objective and collective significance. Regional geography studies the regions, the spaces which present a constitutive homogeneity as in this case the tourism activity. In the case of the country's Cancun-Riviera Maya hinterland region, this area has hosted for more than fifteen years an intense effervescence of the tourism activity, and in many Maya towns of this region, local societies have ventured in tourism as their main or complementary economic activity. The importance of regionalization will be addressed through scale analysis, not only in the study and understanding of alternative tourism, but also through modeling based on the roles stakeholders play in the "turistification" of the rural spaces in a specific region.

Keywords: Regional Geography, Touristic Region, Cancun-Riviera Maya, Touristification, Model.

Fecha de recepción: 22 de febrero de 2019. Fecha de aceptación: 15 de mayo de 2019

CÓMO CITAR: Jouault, S. (2019). Aproximación regional del turismo alternativo: del caso de estudio a la modelización. Dimensiones Turísticas, 3(5), 64-78. https://doi.org/10.47557/MHDC7506 


\section{S. Jouault}

\section{Introducción}

La región turística del traspaís de Cancún-Riviera Maya es heredera de una rica historia prehispánica y colonial que le confiere una carga histórica relevante en el ámbito socioeconómico y cultural. El concepto de traspaís utilizado en esta propuesta se relaciona con las nociones de interior, margen y periferia, y se refiere a la región tierra adentro. En este contexto, es conveniente hacer referencia a algunos temas de estudio relacionados con el concepto de traspaís, desarrollados principalmente en España y Francia en el marco de la geografía del turismo, que contribuyen a la comprensión de las características del traspaís como región tipo (Jouault et al., 2017). En el transcurso de estos últimos veinte años, la región ha sido sede de un número creciente de empresas del sector de la economía social dedicadas al turismo alternativo. Estas empresas invitan al turista a diversificar sus prácticas turísticas hacia la cultura y la naturaleza, a través del descubrimiento del "mundo maya profundo" y de la "exuberante selva tropical".

Si bien el litoral Cancún-Riviera Maya ha sido ampliamente estudiado bajo diferentes enfoques desde hace más de cuarenta años (García de Fuentes, 1979; Dufresne, 1999, Córdoba-Ordoñez y García de Fuentes, 2003; Macías Zapata, 2004 ; Sosa y Jiménez, 2008; Segrado, González, Arroyo y Palafox, 2010; Marín Guardado, 2008 y 2010 ; Monterrubio y Sosa, 2014; Palafox, 2014; Mc Coy y Sosa, 2016; por mencionar solo a algunos estudios), el caso de la región turística emergente del traspaís Cancún-Riviera Maya no ha sido tan abordado desde una perspectiva turística (Daltabuit, Cisneros, Vásquez y Santillán, 2000; Moncada, González, Valtierra y Jiménez, 2011; Moo Canul, 2012, Araujo Santana, 2013; Jouault, 2018).

Partiendo de este contexto, el presente artículo ${ }^{1}$ se inscribe en la necesidad de documentar y analizar el fenómeno turístico en la región, dado su carácter exponencial. También, parece fundamental sistematizar las experiencias entrelazando los diferentes casos con el afán de modelizar el proceso de turistificación de los pueblos del traspaís Cancún-Riviera Maya. Entendemos la turistificación como el proceso por el cual un espacio pasa de ser no-turístico a turístico. Se pretende analizar la importancia de la territorialización del turismo, con el juego de escalas como herramienta básica para comprender el fenómeno turístico en el mundo globalizado, y entendiendo el turismo alternativo como un elemento estructurante para algunas regiones. Después de abordar la importancia de las escalas intermedias y la región como concepto básico de la geografía, la propuesta se enfocará en la modelización de la turistificación de pueblos en la región turística de Cancún-Riviera Maya.

Este trabajo se inscribe en el proyecto de investigación SEP-CONACYT-ANUIES-ECOS Nord 291188 "Desarrollo local y conservación una análisis de los retos de las políticas de lucha contra la pobreza con el turismo alternativo en Yucatán”, en el marco del Acuerdo bilateral México-Francia entre la Escuela Nacional de Estudios Superiores (ENEs Morelia-UnAm) y la Universidad de Angers (Francia). 
La aproximación regional del turismo alternativo: del caso de estudio a la modelización

\section{Antecedentes y metodología}

Esta propuesta tiene por antecedentes la investigación vinculada al Atlas de turismo alternativo en la Península de Yucatán, publicado en 2015 (García de Fuentes et al., 2015). Uno de sus objetivos fue dar a conocer a los actores del sector académico, del sector público y del propio sector social representado, la importancia del sector turístico alternativo en la Península, sus logros, problemas y perspectivas, su forma de interrelación con el sector privado, su mercado, el papel de la política pública y los financiamientos en su desarrollo, entre otros aspectos. El Atlas se elaboró a partir de información primaria recabada directamente en campo, lo que brinda una base única de información para visualizar el fenómeno a escala de una gran región como la Península de Yucatán, donde este tipo de turismo posee gran importancia a causa de su vinculación con el mercado turístico de masas del Caribe mexicano, entre otros factores (García de Fuentes et al., 2015).

Para recabar la información primaria se aplicó un cuestionario de tipo censal integrado por 162 preguntas, del que se obtuvo información -de carácter social, cultural, ambiental y económico- de las 153 empresas turísticas en la Península de Yucatán que operan bajo una lógica de funcionamiento colectivo en escenarios comunitarios. Este mismo cuestionario fue aplicado durante el trabajo de campo realizado entre 2012 y 2016, y se hicieron los análisis correspondientes en el marco de una tesis de doctorado en geografía titulada Mayas de ayer y hoy: el papel de las sociedades locales en el desarrollo turístico de los espacios rurales (Jouault, 2018). Las respuestas a los cuestionarios con cuatro años de intervalo, así como la evolución de la región entre 2012 y 2016, permitieron analizar la relación entre las sociedades locales y la operación de las actividades turísticas en una región denominada traspaís de Cancún-Riviera Maya, a través de las empresas sociales que se dedican al turismo alternativo. Empleamos el sincretismo metodológico, una aproximación tanto cuantitativa como cualitativa, basada en una escala temporal de seis años -entre 2012 y 2018-, que incluyó: revisión bibliográfica en torno a entradas tales como turismo alternativo y región turística; censo de empresas sociales a través de encuestas aplicadas entre 2012 y 2016 en la región del traspaís Cancún-Riviera Maya; elaboración de mapas temáticos a partir de bases de datos organizadas y de sistemas de información geográfica; etnografía, incluyendo observación participativa, realizadas durante una estancia de nueve meses en dos pueblos maya-hablantes. El primero de ellos, Ek Balam, de construcción reciente y cercano al sitio arqueológico homónimo; el segundo, Yokdzonot, ubicado a diez kilómetros de Chichen Itzá. 


\section{La importancia de las escalas intermedias en el juego de escalas}

Así como no se puede entender un lugar sin su región, no es posible entender los efectos del turismo sin la apreciación regional. Se trata, en el sentido más primario, de una relación entre la medida de un objeto real y su representación. Puede asemejarse, en cartografía, a la relación que existe entre una unidad de medida -kilómetro, por ejemplo- y el tamaño que se representa; entre más grande es la escala es posible incluir más territorio, pero a la vez disminuye el detalle que puede visualizarse o representarse. Conforme disminuye el denominador se presenta un territorio más pequeño pero con mucho detalle, hasta llegar a lo local.

En este sentido, "la mundialización es primero un cambio de escalas: la podemos definir como una invención de un espacio a escala mundial a partir de espacios preexistentes a escalas inferiores. Este proceso puede ser comparado a otros, igualmente caracterizados por un cambio de escalas como la construcción de los estados-naciones europeos por agregación de entidades más pequeñas" (Lévy, 2003, p. 638). Y justamente, "el diálogo local-global pasa necesariamente por la consideración de las escalas medias que lo articulan, es decir, utilizando los enfoques regionales que propone la geografía" (Córdoba y García, 2003, p.118). En este sentido, Harvey (2003) plantea uno de los aspectos más complejos para entender el proceso de construcción social del espacio: que este es la combinación del resultado de las acciones humanas sobrepuestas en un lugar a lo largo del tiempo - proceso histórico-, con el resultado de la interacción, en cada momento, de esas acciones a través de una jerarquía de escalas territoriales - proceso geográfico- (Harvey, 2003, p. 95-104).

La construcción social del espacio, y con ello de la diferencia geográfica, como un proceso en el que se combina el resultado de las acciones humanas sobrepuestas en un lugar a lo largo del tiempo -proceso histórico-, con el resultado, en cada momento, de esas acciones a través de una jerarquía de escalas territoriales -proceso geográfico-, es un aspecto ampliamente discutido en el ámbito geográfico y, sin embargo, es también uno de los conceptos más difíciles de incorporar en la práctica, como lo demuestra su ausencia en los marcos de referencia de la planificación y de los ordenamientos, y la manera confusa y contradictoria de introducir en ellos el concepto de región de García y Córdoba (2008, p. 138-139).

La idea de que no es posible pensar un lugar sin la región vinculada puede ejemplificarse con la construcción de Cancún, que no puede desvincularse de la península yucateca. En Yucatán, las décadas de 1970 y 1980 se caracterizaron por el desplazamiento paulatino del eje de acumulación de capital, el cual se orientó del binomio henequén-subsidio al comercio, la construcción y los bienes inmobiliarios; sectores entrelazados que, con un carácter muy especulativo, facilitaron su rápida reproducción.

Determinante en este proceso fue el desarrollo de Cancún. Su construcción fue fundamental para el sostenimiento de la economía de Yucatán, a causa de varios factores: el empleo de obreros de la construcción que remitían su salario a sus familias en la entidad; 
La aproximación regional del turismo alternativo:

del caso de estudio a la modelización

el consumo de materiales de construcción que dinamizó esta industria; el desarrollo de las constructoras yucatecas que abarcaron un importante porcentaje de las obras; los requerimientos de productos y servicios de todo tipo para los que Mérida resultó el centro urbano más cercano capaz de proporcionarlos. La burguesía yucateca participó en la construcción, la venta de materiales para la misma construcción y de productos de primera necesidad; y más tarde estableció en Cancún sucursales de sus comercios (García y Morales, 2000). Por lo tanto, el caso es ilustrativo de la imposibilidad de entender el lugar sin entender primero el contexto regional.

\section{La región, un concepto básico de la geografía}

La región es un segmento diferenciado de la superficie terrestre. Es una de las formas más lógicas y satisfactorias de organizar la información geográfica (Haggett, 1973). Aunque se habla de muchos tipos de región -histórica, natural, nodal, funcional, entre otras-, en este artículo consideramos que existen dos formas de abordar el concepto de región: a) la región como la expresión territorial de una formación social (Lindón y Hiernaux, 2006) que se encuentra en constante movimiento de formación y disolución de acuerdo con los procesos, económicos, demográficos y sociales, y b) la región como un instrumento de análisis, un recorte que hace el investigador de acuerdo con algún criterio y con un fin concreto. La delimitación de las regiones fue un objetivo primordial en épocas pasadas, pero para el análisis actual la delimitación no es un fin en sí mismo, sino una herramienta o método para la comprensión de los fenómenos que se estudian (García y Córdoba, 2008, p. 140-141).

Considerando que en esta primera clasificación intervienen elementos de distinta naturaleza, se propone definir dos tipos de regiones asimilando las propuestas citadas arriba por García y Córdoba (2003): a) región como un espacio construido socialmente a lo largo de la historia, que integra naturaleza y cultura -y por ende a la economía, la demografía, los límites establecidos con fines de control y dominio, entre otros factores-; b) región como un método, un recorte que se hace de acuerdo con un criterio y para un fin concreto: estudio -análisis, investigación académica-, planeación o intervención. Por otra parte, existen dos criterios para definir las regiones en cualquiera de los dos tipos: a) criterio de homogeneidad: es decir, la presencia de un elemento significativo para su identidad, que permite diferenciar este territorio de sus contiguos; puede ser un elemento natural, económico, cultural, e incluso político. Y b) criterio de funcionamiento, que refiere a los vínculos y flujos que se establecen en su interior, entre los puntos que agrupan a la población (desde ciudades hasta localidades más pequeñas), a la economía (infraestructura portuaria, industrial, agrícola, aeropuertos), y a la naturaleza (lagos, bosques, entre otros). Generalmente se parte de un elemento central (polo), una ciudad, un puerto, y se trata de establecer su área de influencia en relación con determinados flujos -personas para obtener servicios médicos o abastecimiento de agua en una cuenca, por ejemplo.

Si bien el concepto de región es fundamental en la geografía, es una noción difícil de aprehender, por una parte, porque a lo largo de la historia se ha empleado con diversas 


\section{S. Jouault}

acepciones. Por otra, porque implica entender el territorio de una manera sintética, es decir, implica abstraer elementos clave que permiten diferenciar un territorio de otro a pesar de sus continuidades. Tanto una región construida socialmente, como el recorte de un espacio con una finalidad específica, se establecen a partir de alguno de los dos criterios anteriores: homogeneidad (áreas definidas por la similitud de algún elemento) o funcionalidad (definida por los flujos que se realizan en su interior). En este contexto, quedan englobados los cuatro tipos de regiones propuestos por Lévy y Lussault (2003), tal como lo muestra el cuadro 1.

\section{Cuadro 1. Tipos de regiones propuestas por Lévy y Lussault}

\begin{tabular}{|c|c|}
\hline Tipos de regiones & Descripción \\
\hline $\begin{array}{l}\text { Región homogénea y } \\
\text { funcional }\end{array}$ & $\begin{array}{l}\text { El nombre refiere a los criterios para establecerla. La categoría parte de los primeros } \\
\text { modelos Christaller (1933) y Lösch (1940), luego Isard (1960) acaba con Boudeville } \\
\text { (1965) y la ciencia regional. }\end{array}$ \\
\hline Región histórica & $\begin{array}{l}\text { Corresponde a un criterio ligado a aquellas regiones fácilmente reconocibles por } \\
\text { su historia natural, cultural, económica, política, o por combinaciones entre estos } \\
\text { elementos -algunas incluso han sido bautizadas a partir de esta historia-. Ejemplos } \\
\text { son la Región de Los Lagos, la Sierra Norte de Puebla, África subsahariana, la región } \\
\text { maya, la región industrial, el Tratado del Atlántico Norte o la Huasteca. Sin embargo, } \\
\text { es preciso apuntar que todo espacio es histórico, y todas las regiones han sido } \\
\text { construidas históricamente. }\end{array}$ \\
\hline $\begin{array}{l}\text { Región económica o } \\
\text { político administrativa }\end{array}$ & $\begin{array}{l}\text { También podría ser cultural, lingüística, gastronómica. Generalmente son regiones } \\
\text { construidas socialmente, pero también pueden ser regiones de planeación, } \\
\text { propuestas, o herramientas para estudiar estos procesos. Predomina el criterio } \\
\text { de homogeneidad, pero en el caso de las económicas también puede partirse } \\
\text { de criterios de funcionalidad. Ejemplos son el Valle del Ruhr en Alemania (región } \\
\text { siderúrgica e industrial) o la Pampa en Argentina (región agropecuaria). }\end{array}$ \\
\hline
\end{tabular}

Región vivida

Es un espacio construido por cada individuo, pueden incluso ser discontinua.

Fuente: Lévy y Lussault, 2003.

La dimensión regional del espacio turístico puede ser explicada por la proximidad existente entre los lugares en los que el turismo es una actividad preponderante. Esta red de sitios turísticos, como la llamaba John Urry (2000), traduce relaciones por líneas visibles o invisibles entre los lugares. La relación entre espacios, actores y prácticas permite el desplazamiento recreativo. Esta red de dimensión regional da origen a la región turística. Y la región turística del traspaís Cancún-Riviera Maya es tanto una región homogénea por sus aspectos bioculturales, una región histórica, la de los mayas cruzoob, una región económica marcada hoy día por la actividad turística y una región vivida por sus habitantes, quienes construyeron espacios de vida en sus pueblos o entre sus pueblos de origen y centros de trabajo. 
La aproximación regional del turismo alternativo:

del caso de estudio a la modelización

\section{El turismo alternativo como elemento estructurante en la construcción de la región del traspaís Cancún-Riviera Maya (C-RM)}

Los resultados del Atlas de turismo alternativo en la Península de Yucatán (García de Fuentes, Jouault y Romero, 2015) mostraron la necesidad de jugar con las escalas, y lo esencial que resulta la aproximación regional para entender las dinámicas de las sociedades locales y su relación con la actividad turística en localidades rurales. El contexto del traspaís de Cancún-Riviera Maya debe ser analizado en una escala local y regional. El estudio de los casos de Yokdzonot y Ek Balam puesto en perspectiva con varios otros pueblos de la región permitirán ilustrar lo planteado hace varios años por Córdoba y García (2003):

Desde la perspectiva espacial el turismo también es agente de desarrollo porque fomenta la modernización y creación de infraestructuras básicas en las que después se apoyarán otras actividades y porque lima las desigualdades regionales en la medida en que ciertas áreas pueden servirse de sus recursos patrimoniales (naturales y culturales) para paliar sus deficiencias de capitalización industrial o agropecuaria (Córdoba y García, 2003, p.119).

Otros autores se han interesado en esta región desde una perspectiva turística; en el 2000, Daltabuit y su equipo pusieron en evidencia que numerosos pueblos no tenían acceso a servicios públicos básicos, tales como el acceso a la educación, la salud, el agua potable, la gestión de aguas usadas y la electricidad (Daltabuit, Cisneros, Vásquez y Santillán, 2000).

Diez años después, Moo Canul (2012) describió y delimitó dicha zona maya -término establecido por antropólogos-, y se insertó en el desarrollo turístico regional. Al mismo tiempo, Moncada y colegas publicaron el libro Tan cerca del Caribe mexicano, tan lejos del turismo (2011). El objetivo del proyecto estribó en medir la importancia del impacto en las comunidades, para las cuales a través del turismo se deseaban disminuir el grado de marginalidad socioeconómica, conservando el balance ecológico y salvaguardando la cultura y la estructura social de los pueblos [...]. El turismo busca generar oportunidades de desarrollo e invertir los fenómenos de migración de una población joven hacia los grandes centros turísticos" (Moncada, González, Valtierra y Jiménez, 2011, p. 10).

En este sentido y para fines de este trabajo, se considera que el turismo alternativo incluye aquellas prácticas que cumplan con los criterios siguientes: alternativas al sistema de desarrollo económico dominante bajo la forma de empresas de la economía social; alternativas al modelo de desarrollo turístico intensivo de sol y playa con prácticas sociales y ambientales más benignas; alternativas a las actividades primarias realizadas en estas localidades (García de Fuentes, Jouault y Romero, 2015).

La región turística del traspaís de Cancún-Riviera Maya se inscribe en el desarrollo regional de la Península de Yucatán y sus diferentes etapas: la creación de Cancún en el principio de los años setenta y la consolidación del destino en los ochenta; la expansión del corredor turístico de la Riviera Maya hacia el sur; y por fin, la turistificación del territorio hacia el interior (cuadro 2). 


\section{S. Jouault}

Cuadro 2. Etapas del desarrollo turístico en el norte de Quintana Roo

\begin{tabular}{|c|c|}
\hline Etapas & Descripción \\
\hline $\begin{array}{l}\text { Boom turístico } \\
\text { de Cancún }\end{array}$ & $\begin{array}{l}\text { En la década de 1970, la organización económica y territorial de la Península se vuelca } \\
\text { hacia un nuevo polo rector: el Centro turístico Integralmente Planeado Cancún, en la } \\
\text { costa de Quintana Roo. Gracias al éxito de este proyecto turístico, Quintana Roo se ha } \\
\text { mantenido entre los estados con las tasas de crecimiento poblacional más altas del país } \\
\text { por varias décadas, y Cancún se ha convertido en un centro urbano de relevancia regional. } \\
\text { Este desarrollo constituye un polo de atracción para la población de las localidades de la } \\
\text { región, cuyos habitantes se emplean convencionalmente en servicios vinculados al turismo } \\
\text { y la construcción. De esta forma, las localidades de interior establecen una relación con } \\
\text { Cancún basada en la oferta de empleo dentro del sector terciario. El auge de Cancún como } \\
\text { centro turístico fortalece la posición de Chichén Itzá como atractivo turístico regional y, } \\
\text { consecuentemente, la ciudad yucateca de Valladolid adquiere un peso turístico vinculado al } \\
\text { tránsito de turistas entre estos dos puntos. }\end{array}$ \\
\hline $\begin{array}{l}\text { Expansión del } \\
\text { corredor turístico } \\
\text { litoral (Riviera } \\
\text { Maya) }\end{array}$ & $\begin{array}{l}\text { En la década de } 1990 \text { comienza la expansión de los proyectos turísticos sobre el litoral de } \\
\text { Quintana Roo, hacia el sur de Cancún. Lo que inicialmente se concibe como un desarrollo } \\
\text { basado en la consolidación de una oferta alternativa al turismo de masas de Cancún, se } \\
\text { materializa como un corredor de grandes complejos hoteleros que funcionan a modo de enclave } \\
\text { turístico. De esta etapa resulta el crecimiento urbano de Playa del Carmen, que se convierte } \\
\text { en un núcleo urbano de importancia regional. }\end{array}$ \\
\hline $\begin{array}{l}\text { El traspaís y los } \\
\text { territorios rurales } \\
\text { de adentro }\end{array}$ & $\begin{array}{l}\text { Auge y expansión del turismo alternativo hacia la región interior de la Península. En la década } \\
\text { del } 2000 \text {, la iniciativa de la Organización Mundial del Turismo (омт) de promoción del turismo } \\
\text { alternativo permea a los esquemas de planeación turística en México; esto, aunado al interés } \\
\text { por fortalecer el componente histórico-cultural en la oferta turística nacional, en la cual las } \\
\text { zonas arqueológicas juegan un papel importante, el turismo rural surge como un eje rector } \\
\text { en las políticas de desarrollo en el país. En este escenario, y con el apoyo de instituciones } \\
\text { gubernamentales y civiles nacionales e internacionales, varias localidades rurales localizadas } \\
\text { en el traspaís del corredor Cancún-Riviera Maya se convirtieron en ofertantes de un turismo } \\
\text { que se presenta como una oferta alternativa y complementaria, estrechamente vinculada a la } \\
\text { dinámica turística del litoral. }\end{array}$ \\
\hline
\end{tabular}

Fuente: Elaboración personal con base en García de Fuentes, Jouault y Romero (2015) y Jouault, García de Fuentes y Jiménez (2017).

La región turística del traspaís Cancún-Riviera Maya engloba a 21 municipios ubicados al norte del estado de Quintana Roo y al oriente del estado de Yucatán. Los límites municipales fueron una base para delimitar esta región, donde la principal vertiente es el corredor litoral de 150 kilómetros entre Cancún y Tulum, y otra es el sitio arqueológico de Chichen Itzá, visitado en 2018 por 2.74 millones de turistas.

Esta región turística, inimaginable sin la cercanía del destino turístico de Cancún-Riviera Maya, se ha estructurado en tres momentos: el primero en los años setenta, cuando numerosos habitantes migraron hacia Cancún y la Riviera Maya para construir y operar 


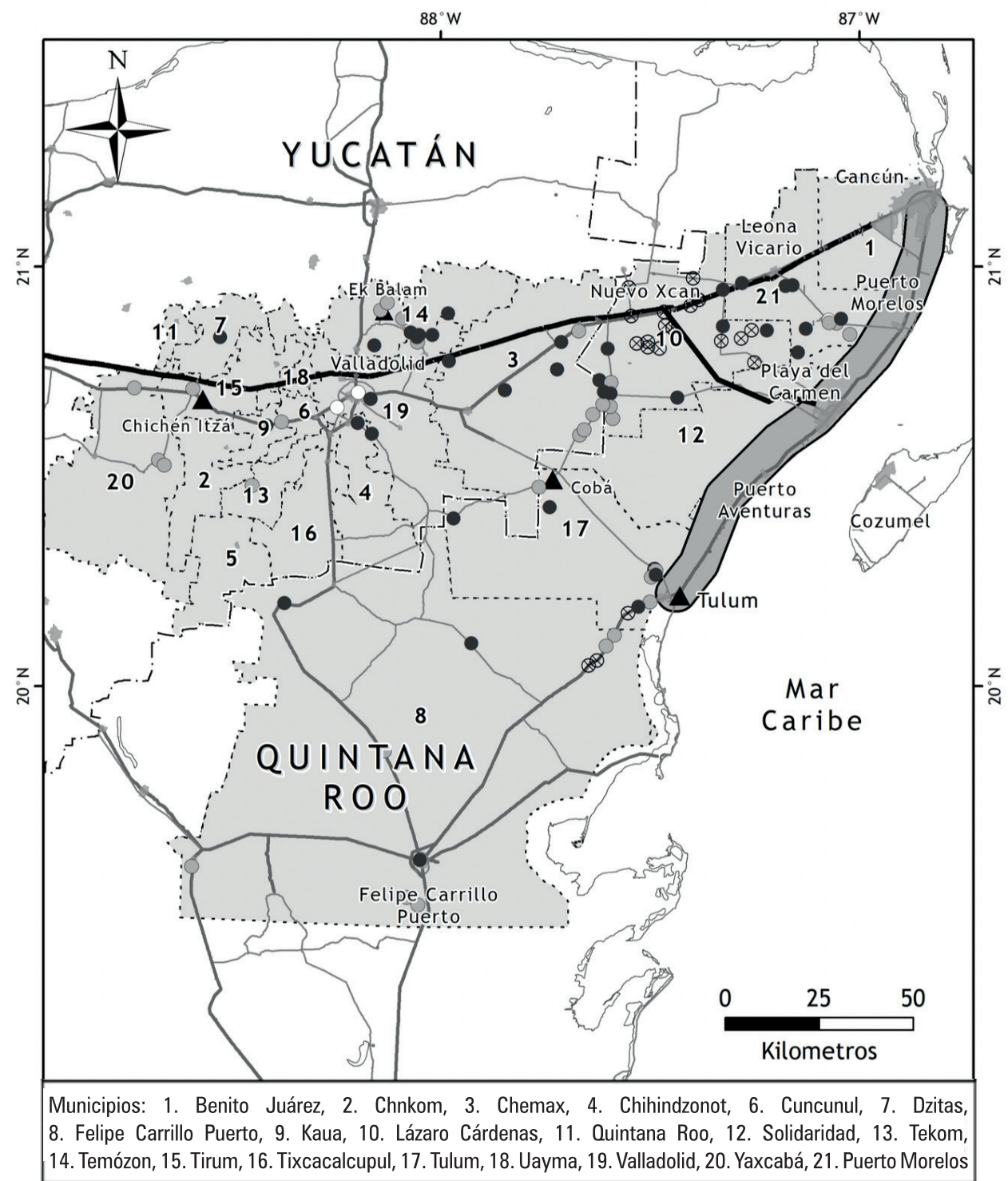

Destinos principales Límites administrativos

Año de inicio de actividades Vías de cumunicación de empresas del sector social

Áreas urbanas

:........ Municipios traspaís

- Antes de 1995

— Carretera federal

Sitios arqueológicos

Riviera Maya

- Entre 1995 y 2010 Carretera estatal

- Después de 2010

$\otimes$ No ha iniciado operaciones

Figura 1. El traspaís Cancún-Riviera maya, una región turística en construcción 


\section{S. Jouault}

infraestructura y equipamientos turísticos; posteriormente, cuando una parte de los insumos consumidos en el litoral -como frutas, hortalizas o carne- comenzaron a ser producidos en la región del traspaís; por fin, un tercer momento contempló una oferta turística alternativa basada en los recursos naturales y la cultura maya, que ha sido desarrollada en pueblos y, de manera general, en los espacios rurales de esta región.

De hecho, a principios de 2016 se contabilizaron noventa empresas de turismo alternativo ubicadas en el traspaís de Cancún-Riviera Maya (operadas por el Estado, privadas y del sector social de la economía), de las cuales setenta eran empresas del sector social; es decir, empresas operadas colectivamente bajo la figura jurídica de sociedad cooperativa, ejido o sociedad de producción rural. El traspaís se ha turistificado de manera exponencial, tal como lo muestra la figura 1. Parte de las veinte empresas que no han comenzado actividades son ejidos que pertenecen a la red forestal de ejidos del municipio de Lázaro Cárdenas (Quintana Roo), los cuales habían solicitado un estudio de factibilidad del turismo en su pueblo.

Esta acelerada turistificación, analizada la escala regional (de ocho a setenta empresas entre 2000 y 2016), se traduce también en flujos turísticos muy importantes hacia algunas empresas (aproximadamente cincuenta mil turistas hacia Pacchén y Tres Reyes, en 2012). Frente a este fenómeno, desplazarse del estudio de caso hacia una sistematización vía la modelización es pertinente para reflexionar en torno al desafío a escala regional.

\section{La construcción de un modelo de la turistificación de pueblos en una región turística}

Si bien es cierto que la gran época de la modelización en geografía y otras ciencias sociales se manifestó durante los años sesenta y setenta, y que hoy día la ola posmodernista detuvo estas tendencias, recurrir a estas herramientas permite distinguir claramente lo particular de lo general. Así, según P. Haggett, “un modelo es una representación esquemática de la realidad elaborada para una demostración” (1973, p. 3). Otros autores tales como Moriconi-Ebrard definen el modelo como "un enunciado teórico fundado en la simulación esquematizada y formalizada en un lenguaje verbal, matemático o gráfico, etc. de un objeto de conocimiento y mas allá de una realidad” (2003, p. 626). Brunet en Les Mots de la Geographie, precisa que el modelo "es una representación formal y depurada de lo real o de un sistema de relaciones" (1992). Se trata entonces de una noción precisa que no puede ser confundida ni con una teoría, ni con un paradigma. Puesto que el modelo esquematiza la realidad y ofrece una imagen cercana a ésta, el modelo posee dos propiedades capitales: representa una pérdida de información en relación con una realidad compleja. Como afirman Chorley y Haggett, "la selectividad implica que los modelos son diferentes de la realidad en el sentido que son solo aproximaciones" (Chorley y Haggett, 1968, p. 444). En contraparte, el modelo simboliza una situación dada que puede ser empleada en situaciones similares. Gracias a su abstracción relativa, es una manera de leer realidades espaciales contrastadas y obtener, entonces, leyes comunes sobre todos los espacios. 
De manera general, mientras más abstracto es un modelo, más evoca una situación general. Como lo afirma P. Haggett, "a cada etapa, perdemos información, el modelo se vuelve más abstracto, pero más general” (Haggett, 1973, p. 4). Dicho de otra manera, modelizar es simplificar y la simplificación es una perdida de información. El proceso de modelización, sin embargo, guarda lo importante de una situación para tener criterios simplificados de diversas situaciones. Así, los modelos tienen grados de abstracción diversos; muchas veces son estáticos, es decir, analizan una situación espacial dada.

A la luz de los resultados del Atlas de turismo alternativo en la Península de Yucatán (García de Fuentes, Jouault y Romero, 2015), años después del estudio del traspaís Cancún-Riviera Maya (Jouault, 2018), y con base en criterios como la tenencia de la tierra, la figura jurídica de la empresa social, el patrimonio turistificado, el acceso a los subsidios, la naturaleza del programa de subsidios o el mecanismo de promoción, proponemos una modelización de la turistificación de pueblos del traspaís Cancún-Riviera Maya. Este modelo fue elaborado con base en algunas evidencias: 78 \% de las empresas sociales que han emergido en el transcurso de los quince últimos años se ubican en la parcela de uso común de un ejido; 75 \% de las empresas sociales son constituidas bajo la forma jurídica de sociedad cooperativa; 78 \% de las empresas sociales han recibido un apoyo del gobierno federal mexicano para implementar la actividad turística en estas localidades rurales; 55 \% de las empresas sociales que se encuentran tierra adentro en Yucatán recibieron financiamiento por parte de la Comisión para el Desarrollo de los Pueblos Indígenas (CDI) a través del Programa de Turismo Alternativo en Zonas Indígenas (PTAZI); ocho de diez empresas sociales cuentan con un mecanismo de promoción hacia el mercado internacional (Jouault, 2018).

El modelo presentado (figura 2), es un esquema simplificado de las interacciones entre actores, permite rendir cuenta de las fuertes tendencias observadas en diferentes pueblos del traspaís Cancún-Riviera Maya. El grupo de ejidatarios que conforma la empresa social bajo diversas figuras jurídicas se encuentra en el corazón del modelo, y entreteje relaciones con el resto del ejido. Es el comisario ejidal, supuestamente orientado por la asamblea ejidal -es decir, la mayoría absoluta o relativa de los ejidatarios-, quien dará luz verde para el uso y el usufructo de los recursos patrimoniales ubicados en el seno del ejido. Muchas veces en acuerdo con la turistificación del patrimonio, el ejido percibirá $10 \%$ de los ingresos brutos vinculados a la actividad turística. Los actores exógenos, tales como los financiadores - programas de subsidios del gobierno federal mexicano- y los consultores, son clave para la comprensión de esta turistificación. El mercado turístico es muchas veces ilusorio por varias empresas sociales. Numerosas empresas reciben una cantidad mínima de turistas. Cabe mencionar que este modelo es válido si los recursos patrimoniales son ubicados en un ejido donde existe el régimen de uso común. Cuando parte o todo el ejido fue parcelado, los recursos patrimoniales ubicados en esta parcela pertenecen al ejidatario o avecindado, y en consecuencia la asamblea ejidal pierde el derecho sobre los usos de dicha parcela. 


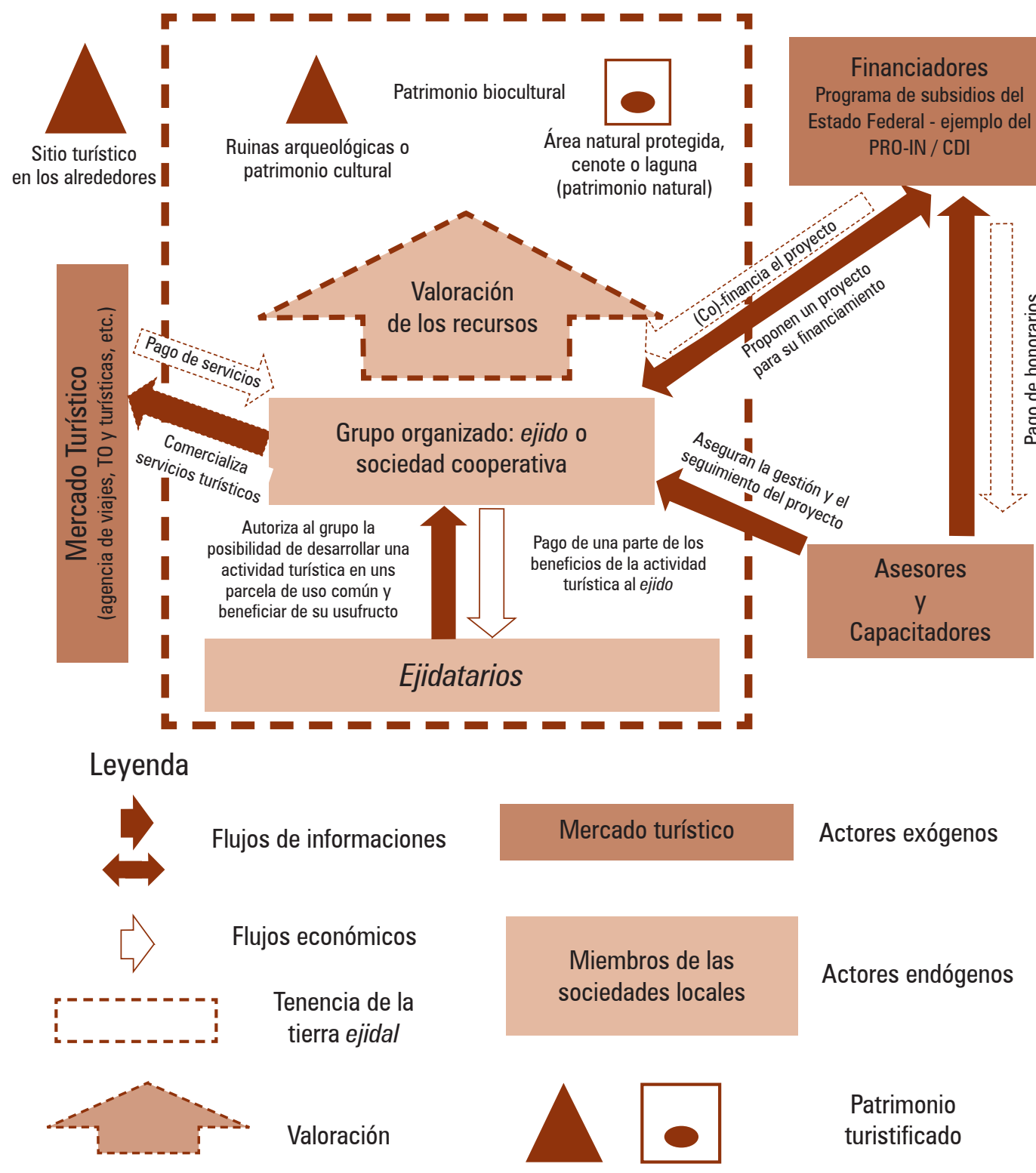

Fuente: Elaboración propia con base en datos extraídos en trabajo de campo.

Figura 2. Modelo de la turistificación de pueblos en la región del traspaís de Cancún-Riviera Maya 
La aproximación regional del turismo alternativo:

del caso de estudio a la modelización

\section{Conclusiones}

La región del traspaís del corredor Cancún-Riviera Maya es actualmente un espacio turistificado, donde la oferta turística se incrementa año tras año, y los flujos turísticos aumentan de manera paralela. El desarrollo de esta región como espacio ofertante de turismo es un fenómeno reciente -que no deja de resultar peculiar en una región que durante la Guerra de Castas refugió a las poblaciones de mayas rebeldes.

La escala regional es una herramienta pertinente de análisis permitiéndonos poner en perspectiva los diferentes casos de estudio teniendo en común un contexto histórico, socioeconómico, biogeográfico y cultural en común. El modelo de turistificación es el resultado de una sistematización diacrónica de las experiencias de turismo alternativo en esta región turística particular. Veinte años después de la creación de las primeras empresas sociales dedicadas al turismo alternativo, impulsadas por programas gubernamentales, el triángulo virtuoso de la turistificación en dicha región del traspáis Cancún-Riviera Maya y otras regiones mexicanas, así como en varios proyectos productivos en el campo mexicano, está basado en la relación tripartita de ejidatarios/consultores-asesores/programas institucionales y subsidiados.

El modelo pone énfasis en las empresas sociales que siguen funcionando a la fecha y alimenta la reflexión según la cual no cualquier lugar puede ser turístico y, principalmente, invita a repensar políticas públicas en torno a la estrategia de turistificación de los espacios rurales mexicanos. Así, en 2015, en la Península de Yucatán, tal como lo mostraron los resultados del Atlas de turismo alternativo (2015), 25 \% de las empresas sociales dedicadas al turismo alternativo habían fracasado y otro $25 \%$ se mantenía bajo una perspectiva paternalista y dependía de un subsidio publico para poder operar (García de Fuentes et al., 2015). Puesto que estos fracasos o casos de paternalismo son inherentes a una planificación turística centralizada, la modelización de la turistificación de pueblos en espacios rurales, resultado de un trabajo de campo minucioso puede ser una herramienta de sistematización para orientar el necesario diseño de las políticas públicas en torno al turismo alternativo de los años venideros.

\section{Referencias}

Araujo Santana, M. R., Parra Vásquez, M. R., Salvatierra Izaba, E. B., Arce Ibarra, A. y Montagnini, F. (2013). Políticas turísticas, actores sociales y ecoturismo en la Península de Yucatán. Economía, Sociedad y Territorio, 13(43), 641-674.

Brunet, R., Ferras, R., Thery H. (1992). Les mots de la géographie: dictionnaire critique, París, Francia: La documentation Française.

Córdoba Ordoñez, J., y García de Fuentes, A. (2003). Turismo, globalización y medio ambiente en el Caribe mexicano. Investigaciones Geográficas. Boletín del Instituto de Geografía UNAM, 52, 117-136. 


\section{S. Jouault}

Chorley, R. J. y Haggett, P. (1968). Models in geography. Londres, Reino Unido: Methuen. Christaller, W. (1933). Die Zentralen orte in Süddeeutschaland. G. Fischer, Jena.

Daltabuit Godas, M., Cisneros H., Vásquez, L. M. y Santillán, E. (2000). Ecoturismo y desarrollo sustentable: impacto en comunidades rurales de la selva maya. Ciudad de México, México: Universidad Nacional Autónoma de México, Centro Regional de Investigaciones Multidisciplinarias.

Dufresne, L. (1999). Les mayas et Cancún. Montréal, Canada: Université de Montréal.

García de Fuentes, A. (1979). Cancún: Turismo y subdesarrollo regional. Ciudad de México, México: unAm-Instituto de Geografía.

García de Fuentes, A y Córdoba y Ordoñez, J. (2008). Desarrollo regional y organización espacial diferenciada: aportes al modelo de ordenamiento territorial mexicano. En Macías Delgadillo, Javier (Coord.), Política territorial en México. Hacia un modelo de desarrollo basado en el territorio. (pp. 97-110 ). Ciudad de México, México: Plaza y Valdéz.

García de Fuentes, A. y Morales, J. (2000). Dinámica regional de Yucatán 1980-2000. Investigaciones Geográficas. Boletín del Instituto de Geografía unam, 42, 157-172.

García de Fuentes, A., Jouault, S. y Romero, D. (2015). Atlas de turismo alternativo de la Península de Yucatán. Mérida, México: Centro de Investigaciones Avanzadas - Unidad Mérida / Facultad de Ciencias Antropológicas, Universidad Autónoma de Yucatán.

Haggett, P. (1973). L'analyse spatiale en géographie humaine. París, Francia: A. Colin.

Harvey, D. (2003). Espacios de esperanza. Madrid, España: Akal.

Jouault, S., García de Fuentes, A. y Jiménez, M. (2017). L'arrière-pays touristique de Cancún Riviera Maya. En N. Bernard, C. Blondy, y P. Duhamel (Coords.), Tourisme, marges et périphéries (pp. 233-252). Rennes, Francia: Presses Universitaires de Rennes.

Jouault, S. (2018). Mayas d'hier et d'aujourd 'hui. Le rôle des sociétés locales dans le développement touristique. Rennes, Francia: Presses Universitaires de Rennes.

Lindón, A. y Hiernaux, D. (2006). La geografía humana: Un camino a recorrer. En D. Hiernaux y A. Lindón (Dirs.), Tratado de geografía humana (pp. 1-22). Barcelona, España: Anthropos / Universidad Autónoma Metropolitana.

Macías Zapata G. (DIR.). (2004), El vacío imaginario. Geopolitica de la ocupación territorial en el Caribe oriental mexicano, Ciudad de México, México: Centro de Investigaciones y Estudios Superiores en Antropología Social.

Marín Guardado, G. (2008). Territorio de resistencia, integración mercantil y producción del espacio turístico en Quintana Roo: trayectorias y transformaciones del mundo maya. En A. Castellanos y J. A. Machuca (Ed.), Turismo, identidades y exclusión (pp. 97-142), Ciudad de México, México: Universidad Autónoma Metropolitana-Iztapalapa / Juan Pablos.

Marín Guardado, G. (2010). Turismo, globalización y mercantilización del espacio y la cultura en la Riviera Maya: un acercamiento a tres escenarios. En R. López (Coord.), Etnia, lengua y territorio. El sureste ante la globalización (pp. 17-56). Ciudad de México: UNAM.

Moncada Jiménez, P., González Neri, I., Valtierra Hernández, A., Jiménez Muñoz, E. (2011). Tan cerca del Caribe mexicano, tan lejos del turismo. Ciudad de México: Ediciones Porrúa / Universidad del Caribe. 
Mc Coy, C., Sosa, P. (2016). Causas y efectos de un destino no sustentable: Caso playas públicas de Cancún, Quintana Roo. El Periplo Sustentable, 31, s/p. Recuperado de: https://rperiplo.uaemex.mx/article/view/4887

Monterrubio, J. C., Josiam, B. M. y Sosa, A. P. (2015). Spring break's social impacts and resident's attitudes in Cancun, Mexico: A qualitative approach. International Journal of Tourism Anthropology, 4(2), 145-16.

Moo Canul M. J., (2012). Lineamientos generales del programa de seguimiento permanente del perfil y grado de satisfacción del turista que visita la zona maya de Quintana Roo. El Periplo sustentable, 23, 143-171.

Moriconi-Ebrard, F. (2003). Modèle. En Levy, J. y Lussault, M. (Dirs.) Dictionnaire de la géographie et de l'espace des sociétés. pp. 626-629. Paris, France : Belin.

Palafox Muñoz, A. (2014). El desarrollo turístico de Quintana Roo desde la perspectiva de la economía política. En T. Cuevas Contreras y A. Palafox Muñoz (Coords.), Complejidad del turismo en la frontera del conocimiento (pp. 203-222). Ciudad Juárez, México: Universidad Autónoma de Ciudad Juárez-Academia Mexicana de Investigación Turística, A.C.

Segrado, R., González, A., Arroyo, L. y Palafox, A. (2010). El desarrollo de Cancún, analizado desde la teoría de los sistemas complejos. Gestión Turística. 14, 9-32.

Sosa, P., Jímenez Martínez, A. (2008). Cocktail Cancún: reflexiones sobre los impactos del turismo en la comunidad local. Cancún, México: Universidad del Caribe.

Urry, J. (2000). Sociology beyond societies. Londres, Reino Unido: Routledge. 